\title{
An Overview of Mobile Ad hoc Network: Application, Challenges and Comparison of Routing Protocols
}

\author{
Sukhpreet Kaur (M.Tech Student) ,Sukhpreet kaur (Asstt. Prof. of CSE Dept) \\ Chandan Sharma (Ph.D. Candidate) \\ Sri Guru Granth Sahib World University Fatehgarh Sahib , \\ Guru Nanak Dev University of ,Amritsar, india (pb.)
}

\begin{abstract}
In the past few years, we have seen a rapid expansion in the field of mobile computing due to the proliferation of inexpensive, widely available wireless devices A mobile ad hoc network (MANET) consists of mobile wireless nodes in which the communication between nodes is carried out without any centralized control. MANET is a self organized and self configurable network where the mobile nodes move arbitrarily. The mobile nodes can receive and forward packets as a router. In this paper provides insight into the potential applications of ad hoc networks and discusses the technological challenges that protocol designers and network developers are faced with. In this paper also present the impact of comparison of different routing protocol in term of different parameters.
\end{abstract}

\section{Introduction}

A wireless network is a growing new technology that will allow users to access services and information electronically, irrespective of their geographic position. Wireless networks can be classified in two types infrastructure network and infrastructure less (ad hoc) networks. Infrastructure network consists of a network with fixed and wired gateways. A mobile host interacts with a bridge in the network (called base station) within its communication radius. The mobile unit can move geographically while it is communicating. When it goes out of range of one base station, it connects with new base station and starts communicating through it. This is called handoff. Recent advancements such as Bluetooth introduced a fresh type of wireless systems which is frequently known as mobile ad-hoc networks. Mobile ad-hoc networks or "short live" networks control in the nonexistence of permanent infrastructure. Mobile ad-hoc network offers quick and horizontal network deployment in conditions where it is not possible.

Ad-hoc is a Latinword, which means "for this or for this only." Early Ad-hoc network only designed for the military application. In 1990's this method will combined with the wireless LAN and Bluetooth. 1997 internet engineering group launched MANET with the routing protocols. They implement routing protocols because to improve the data rate and the efficiency. At present there are more than fifty protocols are implemented in the wireless environment. Mobile ad-hoc network is an autonomous system of mobile nodes connected by wireless links; each node operates as an end system and a router for all other nodes in the network. Mobile ad hoc network is a group of wireless mobile computers (or nodes); in which nodes collaborate by forwarding packets for each other to allow them to communicate outside range of direct wireless transmission. Ad hoc networks require no centralized administration or fixed network infrastructure such as base stations or access points, and can be quickly and inexpensively set up as needed

\section{Advantages of MANET}

There are several advantages of using mobile ad hoc network:-

- Setting up a wireless system is easy and fast and it eliminates the need for pulling out the cables through walls and ceilings.

- Network can be extended to places, which cannot be wired.

- Multiple paths increase reliability.

- Wireless network offers more flexibility and adapt easily to changes in the configuration of the network.

\section{Disadvantages of MANETS}

- Asymmetric links: Most of the wired networks rely on the symmetric links, which are always fixed. But this is not a case with ad-hoc networks as the nodes are mobile and constantly changing their position within network. For example consider a MANET (Mobile Ad-hoc Network) where node B sends a signal to node $\mathrm{A}$ but this does not tell anything about the quality of the connection in the reverse direction. 
- Routing Overhead: In wireless ad hoc networks, nodes often change their location within network. So, some out-of-date routes are generated in the routing table, which leads to unnecessary routing overhead.Interference: This is the major problem with mobile ad-hoc networks as links come and go depending on the transmission characteristics, one transmission might interfere with another one and node might overhear transmissions of other nodes and can corrupt the total transmission.

- Dynamic Topology: This is also the major problem with ad-hoc routing since the topology is not constant. The mobile node might move or medium characteristics might change. In ad-hoc networks routing tables must somehow reflect these changes in topology and routing algorithms have to be adapted. For example in a fixed network routing table updating takes place for every $30 \mathrm{sec}$. This updating frequency might be very low for ad-hoc networks.

\section{Characteristics of Mobile Ad Hoc Networks:-}

Does not rely on a fixed infrastructure for its operation autonomous transitory association of mobile nodes.

- It can be rapidly deployed with user intervention.

- Need not to operate in a standalone fashion but can be attached to the Internet or Cellular networks.

- Devices are free to join or leave the network and they may randomly, possibly

- Resulting in rapid and unpredictable changes.

\section{Working of MANET}

In mobile ad-hoc networks where there is no infrastructure support and since a destination node might be out of range of a source node transmitting packets, a routing procedure is always needed to find a path so as to forward the packets appropriately between the source and the destination. A base station can reach all mobile nodes without routing via broadcast in common wireless networks. In the case of ad-hoc networks, each node must be able to forward data for other nodes.

\section{Applications of MANETS}

\begin{tabular}{|c|c|}
\hline Table 1 & Mobile ad hoc network applications \\
\hline Application & Possible scenarios \\
\hline Tactical networks & $\begin{array}{l}\text { - Military communication and operations } \\
\text { - Automated battlefields }\end{array}$ \\
\hline Emergency services & $\begin{array}{c}\cdot \text { Search and rescue operations } \\
\cdot \text { Disaster recovery } \\
\text { - Replacement of fixed infrastructure in case of environmental } \\
\quad \text { disasters } \\
\cdot \text { Policing and fire fighting } \\
\cdot \text { Supporting doctors and nurses in hospitals }\end{array}$ \\
\hline $\begin{array}{c}\text { Commercial and civilian } \\
\text { Environment }\end{array}$ & $\begin{array}{c}\text { • E-commerce: electronic payments anytime and anywhere } \\
\text { environments } \bullet \text { Business: dynamic database access, mobile offices } \\
\text { - Vehicular services: road or accident guidance, transmission of road } \\
\text { and weather conditions, taxi cab network, inter-vehicle networks } \\
\bullet \text { Sports stadiums, trade fairs, shopping malls } \\
\cdot \text { Networks of visitors at airports }\end{array}$ \\
\hline $\begin{array}{c}\text { Home and enterprises } \\
\text { Networking }\end{array}$ & $\begin{array}{c}\bullet \text { Home/office wireless networking } \\
\bullet \text { Conferences, meeting rooms } \\
\cdot \text { Personal area networks (PAN), Personal networks (PN) } \\
\cdot \text { Networks at construction sites }\end{array}$ \\
\hline Educations & $\begin{array}{c}\cdot \text { Universities and campus settings } \\
\cdot \text { Virtual classrooms } \\
\text { - Ad hoc communications during meetings or lectures }\end{array}$ \\
\hline Entertainment & $\begin{array}{c}\cdot \text { Universities and campus settings } \\
\bullet \text { Virtual classrooms } \\
\text { • Ad hoc communications during meetings or lectures }\end{array}$ \\
\hline Sensor network & $\begin{array}{c}\text { - Home applications: smart sensors and actuators embedded in } \\
\text { consumer electronics } \\
\cdot \text { Body area networks (BAN) } \\
\text { - Data tracking of environmental conditions, animal } \\
\text { movements, chemical/biological detection }\end{array}$ \\
\hline Context aware services & $\begin{array}{c}\text { - Follow-on services: call-forwarding, mobile workspace } \\
\text { - Information services: location specific services, time dependent } \\
\text { services } \\
\text { - Infotainment: touristic information }\end{array}$ \\
\hline
\end{tabular}




\begin{tabular}{|l|c|}
\hline Coverage extension & $\bullet$ Extending cellular network access \\
& $\bullet$ Linking up with the Internet, intranets, etc. \\
\hline
\end{tabular}

\section{Challenges in Mobile Adhoc Network}

Regardless of the attractive applications of MANET introduce several challenges that must be studied carefully before a wide commercial deployment can be expected.

- Routing: Since the topology of the network is constantly changing, the issue of routing packets between any pair of nodes becomes a challenging task. Most protocols should be based on reactive routing instead of proactive. Multi cast routing is another challenge because the multi cast tree is no longer static due to the random movement of nodes within the network. Routes between nodes may potentially contain multiple hops, which is more complex than the single hop communication.

- Security and Reliability: In addition to the common vulnerabilities of wireless connection, an ad hoc network has its particular security problems due to e.g. nasty neighbor relaying packets. The feature of distributed operation requires different schemes of authentication and key management. Further, wireless link characteristics introduce also reliability problems, because of the limited wireless transmission range, the broadcast nature of the wireless medium (e.g. hidden terminal problem), mobility-induced packet losses, and data transmission errors.

- Quality of Service (QoS): Providing different quality of service levels in a constantly changing environment will be a challenge. The inherent stochastic feature of communications quality in a MANET makes it difficult to offer fixed guarantees on the services offered to a device. An adaptive QoS must be implemented over the traditional resource reservation to support the multimedia services.

- Inter-networking: In addition to the communication within an ad hoc network, inter-networking between MANET and fixed networks (mainly IP based) is often expected in many cases. The coexistence of routing protocols in such a mobile device is a challenge for the harmonious mobility management.

- Power Consumption: For most of the light-weight mobile terminals, the communication-related functions should be optimized for lean power consumption. Conservation of power and power-aware routing must be taken into consideration. Multicast: Multicast is desirable to support multiparty wireless communications. Since the multicast tree is no longer static, the multicast routing protocol must be able to cope with mobility including multicast membership dynamics (leave and join).

- Location-aided Routing: Location-aided routing uses positioning information to define associated regions so that the routing is spatially oriented and limited. This is analogous to associatively-oriented and restricted broadcast in ABR.

\section{Routing in Mobile Ad hoc Networks}

Mobile Ad-hoc networks are self-organizing and self-configuring multihop wireless networks, where the structure of the network changes dynamically. In mobile ad-hoc networks there is no infrastructure support as is the case with wireless networks, and since a destination node might be out of range of a source node transferring packets; so there is need of a routing procedure. This is always ready to find a path so as to forward the packets appropriately between the source and the destination. In the case of ad-hoc networks, each node must be able to forward data for other nodes. This creates additional problems along with the problems of dynamic topology which is unpredictable connectivity changes

\section{Problems in routing with Mobile Ad hoc Networks}

- Asymmetric links: Most of the wired networks rely on the symmetric links which are always fixed. But this is not a case with ad-hoc networks as the nodes are mobile and constantly changing their position within network.

- Routing Overhead: In wireless ad hoc networks, nodes often change their location within network. So, some stale routes are generated in the routing table which leads to unnecessary routing overhead.

- Interference: This is the major problem with mobile ad-hoc networks as links come and go depending on the transmission characteristics, one transmission might interfere with another one and no de might overhear transmissions of other nodes and can corrupt the total transmission.

- Dynamic Topology: This is also the major problem with ad-hoc routing since the topology is not constant. The mobile node might move or medium characteristics might change. In ad-hoc networks, routing tables must somehow reject these changes in topology and routing algorithms have to be adapted.

\section{Classification of Routing Protocols in MANET:-}

Routing protocol in MANET are classified into three different categories according to their functionality.

A. Proactive

B. Reactive

C. Hybrid 
Proactive Protocols:- In contrast to table driven routing protocols, on demand compute the route to a specific destination only when needed, so a routing table containing all the nodes as entries does not have to be maintained in each node. When a source wants to send packet to a destination, it invokes a route discovery mechanism to find the path to the destination. The route remains valid till the destination is reachable or until the route is no longer needed.

Table 2

Proactive Routing protocols

\begin{tabular}{|c|c|c|}
\hline Proactive protocols & Predecessor Protocol & Enhancement regarding predecessor \\
\hline DSDR & DBF & Provide single shortest path \\
\hline WRP & DBF & Performance/Count-to-infinity problem \\
\hline FSR & FS & Improved Flooding \\
\hline CGSR & DSDR & Reduces network traffic load \\
\hline
\end{tabular}

Reactive protocols:-These protocols are also called reactive protocols since they don't maintain routing information or routing activity at the network nodes if there is no communication. If a node wants to send a packet to another node then this protocol searches for the route in an on-demand manner and establishes the connection in order to transmit and receive the packet. The route discovery usually occurs by flooding the route request packets throughout the network.

Table: 3

Reactive Routing Protocols

\begin{tabular}{|c|c|c|}
\hline Reactive protocols & Predecessor Protocols & Enhancement regarding predecessor \\
\hline AODV & DSDV & Minimize broadcast message \\
\hline TORA & LMR & Converges quicker in portioned network \\
\hline DSR & -- & -- \\
\hline SSR & ABR & Enhance stability \\
\hline
\end{tabular}

Hybrid protocols: - Since proactive and reactive protocols each work best in oppositely different scenarios, hybrid method uses both. It is used to find a balance between both protocols. Proactive operations are restricted to small domain, whereas, reactive protocols are used for locating nodes outside those domains.

Table 4

Summary of wireless mobile adhoc routing protocols classification

\begin{tabular}{|c|c|c|c|}
\hline & Proactive & Reactive & Hybrid \\
\hline Network organization & Flat / Hierarchical & flat & Both \\
\hline Topology dissemination & periodical & On-demand & Both \\
\hline Route letancy & Always Availble & Available when needed & Medium \\
\hline Communication overhead & High & low & \\
\hline
\end{tabular}

\section{Comparison Of Routing Protocols}

Based on imperative parameters and features of routing protocol, a variety of table-driven (Proactive) routing protocols are compared in Table 5

Table 5

COMPARISON TABLE FOR PROACTIVE ROUTING PROTOCOL

\begin{tabular}{|c|c|c|c|c|}
\hline & DSDV & WRP & FSR & CGSR \\
\hline Route selection & Link state & Shortest path & Shortest path & Shortest path \\
\hline Route & single & single & Single or multiple & Single or multiple \\
\hline Topoloagy structure & flat & flat & hierarachical & Hierarchical \\
\hline Route computation & distributed & distributed & broadcast & Distributed \\
\hline method & broadcast & broadcast & yes & Yes \\
\hline Loop free & yes & Yes, but not & & \\
\end{tabular}


Based on imperative parameters and features of routing protocol, a variety of On Demand (Reactive) routing protocols are compared in Table 6

Table 6

COMPARISON TABLE FOR REACTIVE ROUTING PROTOCOL

\begin{tabular}{|l|l|l|l|l|}
\hline & AODV & TORA & DSR & SSR \\
\hline route selection & $\begin{array}{l}\text { Shortest and } \\
\text { updated path }\end{array}$ & Shortest path & $\begin{array}{l}\text { Shortest and } \\
\text { updated path }\end{array}$ & $\begin{array}{l}\text { Associativity } \\
\text { Stability }\end{array}$ \\
\hline Route & multiple & multiple & multilple & Single \\
\hline Topology & flat & flat & flat & Flat \\
\hline Route computation & Broadcast & Broadcast & Broadcast & Broadcast \\
\hline Method & Unicast & Broadcast & Unicast & Broadcast \\
\hline Loop free & Yes & $\begin{array}{l}\text { NO (not short } \\
\text { lived loop) }\end{array}$ & Yes & Yes \\
\hline
\end{tabular}

\section{Conclusion}

Mobile ad hoc network is decentralised, self-organised, "anytime, anywhere" network and provide cheap communications. In this paper discuss the an overview of different aaplication of the mobile adhoc metwork and various challenges faced by the developer and designer. As there are still many challenges facing wireless ad hoc networks, it is not clear that any particular algorithm or class of algorithm is the best for all scenarios, each protocol has their own merits and demerits and is well suited for certain situations. In this paper also discuss the classification of routing protocols and done comparative analysis for wireless ad hoc networks routing protocols viz. AODV, CGSR, DSR, WRP,DSDV and SSR etc. All the routing protocols are doing good performance in wireless network but reactive protocol doing better performance. Because this reactive protocol path finding protocol perform new path discovery and path maintenance.

\section{References}

[1]. Jeroen Hoebeke, Ingrid Moerman, Bart Dhoedt and Piet Demeester, “An Overview of Mobile Ad Hoc Networks: Applications and Challenges

[2]. Chander Diwaker and Anju Gill "Comparative Analysis of Routing in MANET" International Journal of Advanced Research in Computer Science and Software Engineering, july 2012

[3]. Sankalp Bahadur Singh , Bharat Pesswani “A Performance Analysis Between AODV \& DSR Routing Protocol” IJCTA |July-August 2012

[4]. Anju yadav, "Simulation Study and Implementation on Routing Protocols in MANET" International Journal of Computer Science \& Management Studies, 2012

[5]. P. Misra, "Routing Protocols for Ad Hoc Mobile Wireless Networks", http://www.cis.ohio-state.edu/ jain/cis788-

[6]. Shweta, Vinit Kumar, Anish Mittal, Dr. Pankaj Gupta, Deepak Goel“Analysis of Routing in Mobile Ad-hoc Networks”, International Journal of Computer Science \& Management Studies, 2012

[7]. A.K. Gupta, Dr. H. Sadawarti and Dr. A. K. Verma, "Performance analysis of AODV, DSR \& TORA Routing Protocols" in proceeding of IACSIT International Journal of Engineering and Technology, Vol.2, No.2, April 2010

[8]. Swati Saxena, Birbal Saran, Mr. Vijendra Singh, "SIMULATION AND PERFORMANCE ANALYSIS OF DSDV, OLSR, DSR AND ZRP ROUTING PROTOCOLS IN MANETs", International Journal of Computer Trends and Technology-2012

[9]. C. Siva Ram Murthy and B.S Manoj, "Mobile Ad Hoc Networks- Architectures \& Protocols", Pearson Education, New Delhi, 2004

[10]. Chlamtac, I., Conti, M., and Liu, J. J.-N. Mobile ad hoc networking: imperatives and challenges. Ad Hoc Networks, 1(1), 2003, pp. 13-6.

[11]. HaoYang, Haiyun \& Fan Ye - Security in mobile ad-hoc networks : Challenges and solutions, $\|$, Pg. 3847, Vol 11, issue 1, Feb 2004. 